\title{
Development of INDEL markers to discriminate all genome types rapidly in the genus Oryza
}

\author{
Shinichiro Yamaki ${ }^{1)}$, Hajime Ohyanagi ${ }^{1,2)}$, Masanori Yamasaki ${ }^{3)}$, Mitsugu Eiguchi4), Toshie Miyabayashi ${ }^{4}$, \\ Takahiko Kubo ${ }^{1,5)}$, Nori Kurata ${ }^{1,5)}$ and Ken-Ichi Nonomura*4,5) \\ 1) Plant Genetics Laboratory, National Institute of Genetics, Mishima, Shizuoka 411-8540, Japan \\ 2) Mitsubishi Space Software Co., Ltd., Takezono 1-6-1, Tsukuba, Ibaraki 305-0032, Japan \\ 3) Food Resources Education and Research Center, Graduate School of Agricultural Science, Kobe University, Uzurano1348, Kasai, \\ Hyogo 675-2103, Japan \\ 4) Experimental Farm, National Institute of Genetics, Mishima, Shizuoka 411-8540, Japan \\ 5) Department of Life Science, Graduate University for Advanced Studies (SOKENDAI), Mishima, Shizuoka 411-8540, Japan
}

The wild Oryza species are rich in genetic diversity and are good resources for modern breeding of rice varieties. The reliable ex situ conservation of various genetic resources supports both basic and applied rice research. For this purpose, we developed PCR-based and co-dominant insertion/deletion (INDEL) markers which enable the discrimination of the genome types or species in the genus Oryza. First, 12,107 INDEL candidate sequences were found in the BAC end sequences for 12 Oryza species available in public databases. Next, we designed PCR primers for INDEL-flanking sequences to match the characteristics of each INDEL, based on an assessment of their likelihood to give rise to a single or few PCR products in all 102 wild accessions, covering most Oryza genome types. Then, we selected 22 INDEL markers to discriminate all genome types in the genus Oryza. A phylogenetic tree of 102 wild accessions and two cultivars according to amplicon polymorphisms for the 22 INDEL markers corresponded well to those in previous studies, indicating that the INDEL markers developed in this study were a useful tool to improve the reliability of identification of wild Oryza species in the germplasm stocks.

Key Words: insertion/deletion marker, genome type, genus Oryza, rice, wild species, genetic resource.

\section{Introduction}

Rice (Oryza sativa L.) is one of the most important crops in many countries and so many breeders have made extraordinary efforts to improve yield, grain quality and other agronomical traits in cultivated rice. The completion of the rice genome sequence has promoted molecular breeding and contributed to shorten of the periods for development of new varieties. On the other hand, large scale and long-term cultivation of a few varieties have resulted in a genetic breakdown of modern varieties, for example, in terms of a resistance to biotic stresses (Kottapalli et al. 2010). Wild species are known to be a source of useful genes for potential use in modern rice breeding.

The genus Oryza is composed of 23 species, two cultivated and 21 wild (reviewed by Vaughan and Morishima 2003). On the other hand, regarding chromosome structure, Oryza species are classified into nine genomic types, A to $\mathrm{J}$ (I is absent), according to the pairing affinity of meiotic chromo-

Communicated by Duncan Vaughan

Received January 7, 2013. Accepted April 12, 2013.

*Corresponding author (e-mail: knonomur@nig.ac.jp) somes in hybrid plants. AA wild species are closely related to cultivated rice, $O$. sativa and have been useful resources for current breeding programs (Jena 2010) because of high cross compatibility with cultivars. Non-AA species are distantly related to cultivars and seldom used for breeding, due to lower affinity of the meiotic chromosomes and consequently seed sterility of hybrids with cultivated rice. However, non-AA species contain many agronomically important genes (Jena 2010, Nonomura et al. 2010), so availability of genetically reliable populations of Oryza species would be beneficial in rice breeding.

The ex situ conservation of wild species are always faced with problems arising from outcrossing of species or ecotypes, mixed collections of different species in natural habitats and artificial contamination during conservation. The great efforts by many taxonomists have solved these problems and enabled us to classify and conserve the Oryza species reliably. However, it is still difficult for most researchers to classify the Oryza species based on morphological and physiological traits.

In this study, we aimed to develop new molecular markers for even inexperienced researchers to discriminate species or genome types of wild Oryza accessions easily by 
polymerase chain reaction (PCR) and gel electrophoresis. Single nucleotide polymorphisms (SNPs) and insertion/ deletion (INDEL) markers are the most commonly used markers in plants, because they are easy to use, PCR-based, co-dominant and relatively abundant (Pacurar 2012). Simple sequence repeat (SSR) markers are also useful, but being rapidly replaced with SNP markers, because of the characteristics of SNPs more stable and amenable to automation (reviewed by McCouch et al. 2010). Numerous SNP markers have been developed and used for genomic selection, genomic association and quantitative-trait-loci mapping for inbreeding populations of modern varieties with local varieties or closely-related wild species. In this study, we developed INDEL markers, because they have a merit in easy detection of polymorphims by PCR and direct gel electrophoresis, while SNPs information might be converted into CAPS (cleaved amplified polymorphic sequences) or dCAPS (derived CAPS) and restriction endonuclease cleavage is necessary for SNP detection prior to gel electrophoresis (Michaels and Amasino 1998, Neff et al. 1998). Here, we show that 22 INDEL markers are available to discriminate species or genome types reliably in the genus Oryza.

\section{Materials and Methods}

\section{Plant materials}

All the wild accessions of the genus Oryza were provided by the National Institute of Genetics (NIG) and the National Bioresource Project (NBRP), Japan (Nonomura et al. 2010) and are listed in Table 1. Of 282 wild accessions of the NIG core collection, 42 of rank 1 and 60 of rank 2 were used. The accessions were selected to cover 20 of the 21 wild Oryza species (Fig. 1) and four or more accessions were analyzed for each of most species. In addition, $O$. sativa cv. Nipponbare and cv. Kasalath were used as standard varieties representing ssp. japonica and indica, respectively. All plants were grown in the summer on 2005 and 2006 in a field of the NIG, Mishima, Japan. Genomic DNA was extracted from mature leaves by the CTAB method (Rogers and Bendich 1988).

\section{Identification of INDEL markers}

The bacterial artificial chromosome (BAC)-end sequences (BESs) released by the Oryza Map Alignment Project (OMAP) (Ammiraju et al. 2006, Wing et al. 2005) and available on public databases were used to design PCR primers. The OMAP BESs derived from 12 wild Oryza species (Table 2) were mapped in silico to the reference O. sativa cv. Nipponbare genome (IRGSP Build 5) (http:// rgp.dna.affrc.go.jp/E/IRGSP/Build5/build5.html) by a BLAST search (Altschul et al. 1997). In this step, we selected the BESs that displayed high similarity to the reference sequences except for INDEL regions. For further selection, the gap size was set in the range from 51 to 2,000 base pairs (bp). The primer pair to amplify each of the BESs selected above was designed by Primer3 software (http://primer3. sourceforge.net). The sequences of the reference genome and all primer pairs were put into e-PCR software (http:// www.ncbi.nlm.nih.gov/projects/e-pcr/) and the primer pairs virtually amplifying a single band were selected. Each of the pairs was further screened for giving rise to a single band against its original BES in e-PCR software. For further limitation of the number of candidates, we chose primer pairs that amplified PCR products 50 to $1,500 \mathrm{bp}$ long. In addition, the INDEL size was set at $10 \%$ or more of each PCR product for easy detection of the polymorphism using agarose gel electrophoresis.

\section{Amplicon polymorphism assay}

PCR was performed under conditions of $94^{\circ} \mathrm{C}$ for $2 \mathrm{~min}$ and a subsequent 35 rounds of $94^{\circ} \mathrm{C}$ for $1 \mathrm{~min}, 56^{\circ} \mathrm{C}$ for $1 \mathrm{~min}$ and $72^{\circ} \mathrm{C}$ for $1 \mathrm{~min}$, followed by $72^{\circ} \mathrm{C}$ for $2 \mathrm{~min}$, using GoTaq Green Master Mix kit (Promega) and a T1 Thermocycler (Biometra). Amplicon polymorphisms of the INDEL markers were identified by electrophoresis in 3\% gels of Certified low range ultra agarose (Bio-Rad) followed by ethidium bromide staining.

To detect minute differences in electrophoretic distances more precisely, we also performed fragment analysis of the PCR products using the fluorescent-labeling fragment analyzing system of a capillary sequencer (Applied Biosystems (ABI) PRISM 3130xl) in accordance with the instructions for microsatellite analysis by ABI. ABI PRISM fluorescent primers, fluorescently 5'-labeled with PET, 6-FAM, NED, or VIC dyes, were used for forward INDEL primers. To avoid the "additional A" problem, in which unexpected addition of an adenine residue at the tail of PCR products often makes results unstable, seven nucleotides were artificially added to the $5^{\prime}$ end of reverse primers as recommended by ABI. The PCR product lengths were analyzed using a 3130xl Genetic Analyzer and GeneMapper software (ABI).

\section{DNA sequencing}

PCR products amplified with naked INDEL primer pairs were directly sequenced using a BigDye Terminator v3.1 Cycle Sequencing Kit (ABI) according to the manufacturer's instructions. Some products were cloned into vector pCRII (Invitrogen), then amplified and sequenced with universal M13 forward and reverse primers.

\section{Phylogenetic analysis}

A rooted phylogenetic tree was drawn by the UPGMA method (Sokal and Michener 1958) on the basis of the PCR product lengths of INDEL markers determined manually following gel electrophoresis. Data from the fragment analysis were used only to judge the size of DNA fragments showing similar, but slightly different mobilities in the gel. The tree construction was based on Nei's chord distance (Nei et al. 1983) and performed using Populations version 1.2.31 software (http://bioinformatics.org/ tryphon/ populations/) with 1,000 bootstraps. 
Table 1. The accessions of wild Oryza species used in this study

\begin{tabular}{|c|c|c|c|c|c|c|c|}
\hline Acc No. & Species & Genome type & Origin & Acc No. & Species & Genome type & Distribution \\
\hline W0106 & rufipogon & AA & India & W1361 & officinalis & $\mathrm{CC}$ & Malaysia \\
\hline W0120 & rufipogon & AA & India & W1830 & officinalis & $\mathrm{CC}$ & unknown \\
\hline W1294 & rufipogon & AA & Philippines & W1131 & officinalis & $\mathrm{CC}$ & India \\
\hline W1866 & rufipogon & AA & Thailand & W1302 & officinalis & $\mathrm{CC}$ & Philippines \\
\hline W1921 & rufipogon & AA & Thailand & W1814 & officinalis & $\mathrm{CC}$ & Sri Lanka \\
\hline W2003 & rufipogon & AA & India & W1805 & rhizomatis & $\mathrm{CC}$ & Sri Lanka \\
\hline W0630 & rufipogon & AA & Myanmar & W1527 & eichingeri & $\mathrm{CC}$ & Uganda \\
\hline W1236 & rufipogon & $\mathrm{AA}$ & unknown & W1519 & eichingeri & $\mathrm{CC}$ & Uganda \\
\hline W1807 & rufipogon & AA & Sri Lanka & W1522 & eichingeri & $\mathrm{CC}$ & Uganda \\
\hline W1945 & rufipogon & $\mathrm{AA}$ & unknown & W1525 & eichingeri & $\mathrm{CC}$ & Uganda \\
\hline W2051 & rufipogon & AA & unknown & W1166 & latifolia & CCDD & Mexico \\
\hline W2078 & rufipogon & AA & Australia & W1197 & latifolia & CCDD & Colombia \\
\hline W2263 & rufipogon & AA & Cambodia & W2200 & latifolia & CCDD & Brazil \\
\hline W0652 & barthii & AA & Sierra Leone & W0019 & latifolia & CCDD & Cuba \\
\hline W1588 & barthii & AA & Cameroun & W1181 & latifolia & CCDD & Panama \\
\hline W0698 & barthii & AA & Guinea & W0017 & alta & CCDD & Surinam \\
\hline W0720 & barthii & AA & Mali & W1182 & alta/latifolia & CCDD & Guyana \\
\hline W0747 & barthii & AA & Mali & W0018 & alta & CCDD & Paraguay \\
\hline W1646 & barthii & AA & Tanzania & W1147 & alta & CCDD & Surinam \\
\hline W1169 & glumaepatula & $\mathrm{AA}$ & Cuba & W0613 & grandiglumis & CCDD & Brazil \\
\hline W2145 & glumaepatula & AA & Brazil & W1194 & grandiglumis & CCDD & Brazil \\
\hline W2199 & glumaepatula & AA & Brazil & W2220 & grandiglumis & CCDD & Brazil \\
\hline W1185 & glumaepatula & AA & Suriname & W1476 & grandiglumis & CCDD & Brazil \\
\hline W1187 & glumaepatula & AA & Brazil & W1480(B) & grandiglumis & CCDD & Brazil \\
\hline W1196 & glumaepatula & AA & Colombia & W0008 & australiensis & EE & Australia \\
\hline W1625 & meridionalis & AA & Australia & W1628 & australiensis & $\mathrm{EE}$ & Australia \\
\hline W1635 & meridionalis & AA & Australia & W1632 & australiensis & $\mathrm{EE}$ & Australia \\
\hline W1297 & meridionalis & AA & Australia & W2086 & australiensis & $\mathrm{EE}$ & Australia \\
\hline W1627 & meridionalis & AA & Australia & W2104 & australiensis & $\mathrm{EE}$ & Australia \\
\hline W2069 & meridionalis & AA & Australia & W1401 & brachyantha & $\mathrm{FF}$ & Sierra Leone \\
\hline W2079 & meridionalis & AA & Australia & W1711 & brachyantha & FF & Cameroun \\
\hline W2103 & meridionalis & $\mathrm{AA}$ & Australia & W1407(B) & brachyantha & $\mathrm{FF}$ & Mali \\
\hline W1413 & longistaminata & AA & Sierra Leone & W1706 & brachyantha & $\mathrm{FF}$ & Chad \\
\hline W1508 & longistaminata & AA & Madagascar & W0003 & granulata & GG & India \\
\hline W0643 & longistaminata & AA & Gambia & W0067(B) & granulata & GG & Thailand \\
\hline W0708 & longistaminata & AA & Guinea & W0005 & granulata & GG & Ceylon \\
\hline W1540 & longistaminata & AA & Congo & W0615 & granulata & GG & Myanmar \\
\hline W1624 & longistaminata & AA & Cameroun & W1356 & meyeriana & GG & Malaysia \\
\hline W1514 & $\operatorname{punctata}(2 x)$ & BB & Kenya & W1348 & meyeriana & GG & Malaysia \\
\hline W1590 & $\operatorname{punctata}(2 x)$ & BB & Cameroun & W1352 & meyeriana & GG & Malaysia \\
\hline W1024 & punctata $(4 x)$ & $\mathrm{BBCC}$ & Ghana & W1354 & meyeriana & GG & Malaysia \\
\hline W1408 & punctata $(4 x)$ & $\mathrm{BBCC}$ & Nigeria & W1360 & meyeriana & GG & Malaysia \\
\hline W1474(B) & punctata(4x) & $\mathrm{BBCC}$ & Chad & W2068 & meyeriana & GG & unknown \\
\hline W1213 & minuta & $\mathrm{BBCC}$ & Philippines & W0001 & ridleyi & HНJJ & Thailand \\
\hline W1331 & minuta & $\mathrm{BBCC}$ & Philippines & W0604 & ridleyi & HНJJ & Malaya \\
\hline W0016 & minuta & $\mathrm{BBCC}$ & Surinam & W2033 & ridleyi & HHJJ & Thailand \\
\hline W1319 & minuta & $\mathrm{BBCC}$ & Philippines & W2035 & ridleyi & HHJJ & Thailand \\
\hline W1323 & minuta & $\mathrm{BBCC}$ & Philippines & W1220 & longiglumis & HНJJ & Indonesia \\
\hline W1328 & minuta & $\mathrm{BBCC}$ & Philippines & W1215 & longiglumis & HHJJ & Indonesia \\
\hline W1329 & minuta & $\mathrm{BBCC}$ & Philippines & W1224 & longiglumis & HНJJ & Indonesia \\
\hline W0002 & officinalis & $\mathrm{CC}$ & Thailand & W1228 & longiglumis & HНJJ & Indonesia \\
\hline
\end{tabular}

Flow cytometry

In addition to wild species, we used a haploid plant, produced from $O$. sativa $\mathrm{cv}$. Nipponbare by the slightly modified method of the anther culture described by Niizeki and Oono (1968), to normalize the nuclear DNA contents of wild species. The preparation of samples and flow cytometric analysis was performed in accordance with Miyabayashi et al. (2007) with slight modifications. The sample nuclei were extracted from a piece of adult leaves $(5 \times 5 \mathrm{~mm})$. The leaves were chopped with a razor blade in $400 \mu \mathrm{L}$ of extraction 


\begin{tabular}{|c|c|c|c|c|c|c|c|c|c|c|c|c|c|c|c|c|c|c|c|c|}
\hline \multirow{3}{*}{ Marker ID } & \multicolumn{20}{|c|}{ wild Oryza species } \\
\hline & \multicolumn{5}{|c|}{$\mathrm{AA}$} & \multirow{2}{*}{$\begin{array}{l}\text { BB } \\
\text { pun }\end{array}$} & \multicolumn{2}{|c|}{$\mathrm{BBCC}$} & \multicolumn{3}{|c|}{$\mathrm{CC}$} & \multicolumn{3}{|c|}{ CCDD } & \multirow{2}{*}{$\begin{array}{l}\mathrm{EE} \\
\text { aus }\end{array}$} & \multirow{2}{*}{$\begin{array}{l}\text { FF } \\
\text { bra }\end{array}$} & \multicolumn{2}{|c|}{ GG } & \multicolumn{2}{|c|}{ HHJJ } \\
\hline & ruf & bar & glu & mer & lon & & pun & $\min$ & off & eic & rhi & lat & alt & gra & & & gta & mey & rid & $\log$ \\
\hline Ch01-301W & 352 & & & 375 & & 410 & & & & & & & & & & & & & $296 / 4$ & 410 \\
\hline Ch02-269W & 259 & & & 285 & & 124 & & & & & & & & & & & 115 & & 120 & \\
\hline Ch02-308W & 112 & & & 160 & & 176 & & & & & & & & & & & & & & \\
\hline $\mathrm{Ch} 02-342 \mathrm{~W}$ & 209 & & & & & & & & & & & & & & & 405 & & & & \\
\hline Ch02-343W & 380 & & & $\begin{array}{l}210, \\
370\end{array}$ & $\begin{array}{l}135^{*} \\
210^{*}\end{array}$ & 135 & & & & & & & & & & & 140 & & $120 / 1$ & \\
\hline Ch03-128W & 298 & & & & & 280 & & & & & & & & & & & 420 & & $222 / 2$ & \\
\hline Ch03-173W & 369 & & & 130 & & & & & & & & & & & 137 & & 153 & & 135 & \\
\hline Ch03-363W & 461 & & & & & & & & 550 & & & & & & & 363 & & & $430 / 4$ & \\
\hline Ch04-276G & 132 & & & & & & & & & & & & & & & 150 & 360 & & 150 & \\
\hline Ch04-312W & 178 & & & & 200 & & & & & & & $160 *$ & $160:$ & & 160 & 396 & & & & \\
\hline Ch05-067W & 195 & & & & & 145 & & & & & & & & & & & & & 135 & \\
\hline Ch05-070W & 263 & & & & & & & & & & & $371 *$ & $\begin{array}{l}263^{*} \\
371^{*}\end{array}$ & 371 & 365 & 310 & 320 & & 336 & \\
\hline Ch05-109G & 398 & & & & & 180 & & & & & & & & & 500 & & 122 & & & \\
\hline Ch05-202W & 148 & & & & & & & & & & & & & & & 130 & 65 & & & \\
\hline Ch05-277W & 326 & & & & & & & & & & & & & & & & & & 362 & \\
\hline Ch06-269W & 299 & & & 600 & & 357 & & & & & & & & & & & & & & \\
\hline Ch06-300W & 180 & & & & & & & & & & & & & & & 210 & 360 & & $180 *$ & \\
\hline Ch06-306W & 124 & & & & & & $185 *$ & & 182 & & & & & & & 140 & 120 & & 145 & 150 \\
\hline Ch07-233W & 281 & & & 440 & & 200 & & & 300 & $300 \%$ & & $281 *$ & $281 \%$ & & & & & & $181 /$ & 1350 \\
\hline Ch08-006W & 234 & & & & 270 & & & & & & & & & & & 372 & 245 & & 410 & \\
\hline Ch09-037G & 161 & & & & & & & & & & & & & & & 140 & 363 & & 330 & \\
\hline Ch10-044G & 121 & & & & & & & & & & & & & & & 150 & 553 & & & \\
\hline
\end{tabular}

Fig. 1. A diagram of the polymorphic PCR band pattern of 20 wild Oryza species obtained using 22 INDEL markers. The color pattern indicates how many polymorphic bands were detected in each of 22 INDEL markers for 20 wild Oryza species. The species indicated with a same color exhibited an identical band size for each marker on the gel electrophoresis. The number in each color indicates the estimated band size (bp). For example, the Ch7-233W marker gave rise to 5 different bands: 281 bp (magenta), 440 bp (yellow), $200 \mathrm{bp}$ (green), $300 \mathrm{bp}$ (light blue) and $181 /$ $350 \mathrm{bp}$ (dark blue). In BBCC and CCDD tetraploid species, this marker gave two bands from both BB and CC types, colored green and light blue. Two numbers with a slash (ex., 181/350) indicate that in tetraploid species (for example, HHJJ), the genome types from which both bands were raised are uncertain. Two numbers with a comma (ex., 210, 370) indicate that the marker amplifies two PCR bands in a diploid species. The numbers with asterisks indicate that those bands are polymorphic in several accessions within the same species. ruf, rufipogon; bar, barthii; glu, glumaepatula; mer, meridionalis; lon, longistaminata; pun, punctata; min, minuta; off, officinalis; eic, eichingeri; rhi, rhizomatis; lat, latifolia; alt, alta; gra, grandiglumis; aus, australiensis; bra, brachyantha; gta, granulata; mey, meyeriana; rid, ridleyi; log, longiglumis. The seven marker IDs colored red are a minimum marker set useful for classifying species and genome species in the genus Oryza.

solution A (Partec) and incubated for $30 \mathrm{~min}$. The supernatant was filtered with $50 \mu \mathrm{m}$ - and subsequently with $20 \mu \mathrm{m}$ CellTrics filters. $160 \mu \mathrm{L}$ of staining solution B (Partec) was added to the supernatant and incubated for $30 \mathrm{~min}$. The extract from the wild species sample was mixed with an equal volume of that from the haploid plant and supplied for the measurement of nuclear DNA contents by ploidy analyzer PA system (Partec) according to the manufacturer's instruction.

\section{Online disclosure of marker information}

Information on the 22 INDEL markers obtained in this study is open access on the integrated rice science database,
Oryzabase (Yamazaki et al. 2010, http://www.shigen.nig.ac. jp/rice/oryzabaseV4/).

\section{Results}

Selection of INDEL markers to discriminate wild Oryza species

To design PCR primers for discriminating wild relatives of rice, BESs derived from twelve wild species, including $O$. nivara and $O$. coarctata in accordance with the taxonomy in Ammiraju et al. (2006), were selected from public databases and compared to the reference $O$. sativa cv. Nipponbare genome. This permitted the identification of 
Table 2. The number of putative INDELs detected in silico in this study

\begin{tabular}{lccr}
\hline \hline Oryza species* & $\begin{array}{c}\text { Genome } \\
\text { type }\end{array}$ & $\begin{array}{c}\text { No. INDELs } \\
\text { candidate sites } \\
\text { in this study }\end{array}$ & $\begin{array}{c}\text { No. BESs used } \\
\text { in this study }\end{array}$ \\
\hline rufipogon & AA & 439 & 70,982 \\
nivara & AA & 462 & 106,124 \\
glaberrima & AA & 556 & 66,821 \\
punctata & BB & 1,270 & 68,384 \\
minuta & BBCC & 1,911 & 169,460 \\
officinalis & CC & 1,504 & 101,091 \\
alta & CCDD & 1,370 & 128,732 \\
australiensis & EE & 705 & 135,769 \\
brachyantha & FF & 550 & 67,364 \\
granulata & GG & 538 & 138,171 \\
ridleyi & HHJJ & 831 & 204,729 \\
coarctata & HHKK & 1,971 & 195,285 \\
\hline & & 12,107 & $1,452,912$ \\
\hline
\end{tabular}

* The taxonomy is in accordance with Ammiraju et al. (2006)

12,107 putative INDELs in the genomes of wild species compared with cultivated rice (Table 2). 3,244 INDEL loci were shared by 3 to 10 species each (referred to hereafter as multi-INDELs) (data not shown). The multi-INDELs were further reduced to 120 loci based on the degree of conservation of INDEL-flanking sequences and examination of the lengths of PCR products (see Methods). In addition, we selected 104 INDELs, each of which was detected with reference to a single BES from O. rufipogon, O. punctata, $O$. brachyantha, or $O$. granulata (single INDEL). In total, 224 markers were investigated for their applicability in classification of wild rice relatives. A total of 40 rice microsatellite (RM) markers (McCouch et al. 2002) were also investigated. However, all of the RM markers gave intraspecifically polymorphic PCR fragments only in AA-genome species (data not shown); therefore, they were excluded from subsequent analyses.

A total of 22 wild accessions representing the 20 Oryza species in addition to two rice varieties, $O$. sativa ssp. japonica $\mathrm{cv}$. Nipponbare and ssp. indica $\mathrm{cv}$. Kasalath, were used for the first assessment of INDEL markers. We screened the 224 primer sets, focusing on their ability to amplify PCR products in all genome types, especially in distantly related EE, FF, GG or HHJJ species, since this study aimed to establish PCR-based markers applicable for all Oryza species. In this screening, 60 of 120 multi- and 13 of 104 single INDELs were selected and further analyzed (Supplemental Fig. 1). More efficient amplification in distantly related species by multi-INDELs than by single INDELs suggested that INDEL-flanking sequences are diverse among species, and that careful mining of flanking sequences conserved across multiple species is important for efficient selection of INDEL markers. In the second assessment, 22 out of 73 INDELs that gave clear PCR bands in 102 wild accessions were selected (Table 3 and Supplemental Fig. 1) and further analyzed. The 22 INDEL loci were widely distributed on all Nipponbare chromosomes except for chromosomes 11 and

Table 3. The 22 INDEL markers developed to discriminate wild Oryza species in this study

\begin{tabular}{|c|c|c|c|c|}
\hline \multirow{2}{*}{ Marker ID* } & \multicolumn{2}{|c|}{ Primer sequences } & \multirow{2}{*}{$\begin{array}{c}\text { INDEL } \\
\text { size (bp) }\end{array}$} & \multirow{2}{*}{ BES ID } \\
\hline & Forward & Reverse & & \\
\hline Ch01-301W & TTTGTTCATCTGCATCAACTCA & TGATCATACGATGGAAAGGTAGA & 56 & OR_ABa0229J12.r \\
\hline Ch02-269W & TTCGGTAAAGAACCTCTTGAGTG & GATTCTAGTGCCATTTCGCC & 135 & $\mathrm{OP}^{-} \mathrm{Ba} 0011 \mathrm{~A} 10 . \mathrm{r}$ \\
\hline Ch02-308W & CCTTAAGAAATTGTTAGTTCAGGCA & СTTCTTCTTGCTAGCAGTTGTCT & 64 & $\mathrm{OP} \quad \mathrm{Ba} 0008 \mathrm{P} 06 . \mathrm{f}$ \\
\hline Ch02-342W & TGGCAGACCATCTGAGAGAG & CCAGAAATCAGCAATCTGCAA & 196 & $\mathrm{OB} \_\mathrm{Ba} 0075 \mathrm{C} 24 . \mathrm{f}$ \\
\hline Ch02-343W & TTCTCCACCCTCGTCTTCTC & TTGCTCTCCAGCTTCTCCTC & 245 & $\mathrm{OB} \_\mathrm{Ba} 0060 \mathrm{I} 13 . \mathrm{r}$ \\
\hline Ch03-128W & TGATTCCTTGGTAGTCTTCCC & TGCTCACCATAGACTCTTCCA & 76 & OR_ABa0265L24.r \\
\hline Ch03-173W & AGGCAAAGTTCAGAATGCAA & AGCGGCAATAGCCATCTAAG & 217 & $\mathrm{OG}_{-} \mathrm{ABa} 0011 \mathrm{~K} 22 . \mathrm{r}$ \\
\hline Ch03-363W & TTTCCGTCAGATTGCACATT & TGATTTACCACCAAACAGTAAGTCA & 98 & $\mathrm{OB}^{-} \mathrm{Ba} \mathrm{B} 073 \mathrm{~N} 03 . \mathrm{f}$ \\
\hline Ch04-276G & GGTACCTCCAGGAATCCCAT & CCAATGTGCATGGCATTTAG & 229 & OG_ABa0018K10.r \\
\hline Ch04-312W & TTCTTTGTCGTGATCGCAAG & TTTCATTCAACGTGGTGGTT & 218 & OR_ABa0207K04.f \\
\hline Ch05-067W & CCCATTCCCTATACCTGTGTAAA & AGAATCACAGAGGATCCGAA & 60 & OR_ABa0151A19.r \\
\hline Ch05-070W & GGAAGAAAGCAAGGATGCAA & TCTGCTGTCATATGCTTGGG & 108 & OA_BBa0041D18.f \\
\hline Ch05-109G & TGATGATGAAATACCTTGCCC & TGTATGGCTGCATTTGCACT & 276 & $\mathrm{OG}_{-} \mathrm{ABa} 0128 \mathrm{~B} 15 . \mathrm{f}$ \\
\hline Ch05-202W & TCTTCAAGAAACCAGAAGATCTGA & TGGATGTGCTTCTGACGCTA & 83 & OG_ABa0074B07.r \\
\hline Ch05-277W & CCAGAACCGTTGTTCCTGTT & GGATGTTGAGAAGGGTGGAA & 64 & OR_ABa0019H23.r \\
\hline Ch06-269W & CCAATGAAATGCAGTCGAGA & GGACACATTCAACCCTCACA & 79 & $\mathrm{OC}^{-} \mathrm{Ba} 0241 \mathrm{E} 15 . \mathrm{f}$ \\
\hline Ch06-300W & CACAGACAGTGTCCAGAGTTCAG & GCCTTGAAAGTTGAAACCCA & 180 & OG_ABa0049K22.f \\
\hline Ch06-306W & GAGCCCTCGGTTAGATGTGA & CGTGCCGTATATGTCCTGAA & 61 & $\mathrm{OM}^{-} \quad \mathrm{Ba} 0207 \mathrm{M} 10 . \mathrm{f}$ \\
\hline Ch07-233W & TCACCAAGCCAATTCTTCTTC & ССТССТАAACCAGACTGCACA & 100 & OR_ABa0269N20.f \\
\hline Ch08-006W & TTGCATTGAATCAGTAGGTCAA & AGGACCTTGATTTGCCATGT & 138 & $\mathrm{OB}_{-} \_\mathrm{Ba} 0054 \mathrm{E} 21 . \mathrm{f}$ \\
\hline Ch09-037G & CCGGAGTCCTATCCACAGGT & TTGGGCATCACCTGATAAGA & 202 & OG_ABa0001G03.f \\
\hline Ch10-044G & CTTGTTTCCAGCAAGGTTGG & TACCAGGTTTGCTGCATGTT & 432 & OG_ABa0068J15.r \\
\hline
\end{tabular}

* The suffixes $\mathrm{W}$ and $\mathrm{G}$ indicate that the markers are multi- and single (granulata)-INDELs, respectively. 
12 and eighteen of them were multi-INDELs (suffixed "W" in Table 3). The size of the 22 INDELs ranged from 56 to $432 \mathrm{bp}$ and averaged $150.8 \mathrm{bp}$ (Table 3 ).

Validation of 22 INDEL markers in discrimination of wild Oryza species

Each of the 22 selected INDEL markers exhibited various interspecific amplicon polymorphisms in the genus Oryza (Fig. 1). To validate the reliability of these markers for discriminating Oryza species, they were applied to phylogenetic analysis of 102 wild accessions and two cultivars (Fig. 2). The band sizes of PCR products were manually determined following agarose gel electrophoresis. In addition, for detecting minute differences in electrophoretic distances more precisely, we attempted fragment analysis. However, it was difficult to completely replace the manual method by fragment analysis, because the size of amplicons varied even among wild accessions from the same species. This may be due to numerous inter- and intraspecific SNPs and small INDELs in the sequences flanking the targeted INDEL being responsible for minute differences in electrophoretic distances. Thus, we decided to evaluate PCR band sizes mainly manually, using fragment analysis to support the manual method. All raw data obtained by either method are compiled in Supplemental Table 1. Furthermore, DNA sequencing confirmed that each of the 22 INDEL primer sets enabled amplifying PCR products from a syntenic locus shared by multiple genome types of Oryza species (an example of sequence alignments is shown in Supplemental Fig. 2), indicating the reliability of these markers. Only one of the 22 INDELs was derived from an exonic region (Ch04-276G), four were from intergenic regions (Ch04-312W, Ch05109G, Ch05-202W and Ch06-300W) and all remaining INDELs were from intronic regions (data not shown).

\section{Phylogenetic analysis}

Phylogenetic analysis using the 22 INDEL markers selected above successfully divided 102 wild accessions into 9 genome groups (Fig. 2). Here, the taxonomy of the genus Oryza depended on Vaughan and Morishima (2003). Thirtyeight accessions of AA-genome species were divided into 3 phylogenetic groups: the group including rufipogon, barthii and glumaepatula, the meridionalis group and the longistaminata group. Thirty-seven accessions of BB, CC, BBCC and CCDD genome species, the "officinalis complex" (see below), were divided into five independent but closely related groups (Fig. 2). Fourteen accessions of $O$. latifolia, $O$. alta and $O$. grandiglumis, all classified into the CCDD tetraploids, were divided into 4 closely-related groups (Fig. 2). The accessions of latifolia and/or alta segregated into all 4 groups. All $O$. grandiglumis accessions were in a single group with two accessions of alta or latifolia (Fig. 2). The officinalis complex groups was first connected with EE-genome species and then with the AA-genome in the phylogenetic tree (Fig. 2). In contrast, GG, FF and HHJJ taxa were distant to AA taxa (Fig. 2).
A minimum set of INDELs to discriminate 9 genome types

This study revealed that seven INDEL markers (colored red in Fig. 1) were sufficient to discriminate 9 genome types and several species in the genus Oryza.

Four INDELs enabled classification of wild species into each genome group by PCR and agarose gel electrophoresis (Fig. 1 and Supplemental Fig. 1). First, Ch06-306W gave PCR bands characteristic of the CC, FF, GG and HHJJ genome groups. HHJJ species were separable into $O$. ridley and $O$. longiglumis, allowing consequent determination of $O$. brachyantha, since the FF genome is composed of a single species. Second, Ch07-233W characterized diploid O. punctata (BB), diploid CC genome species and tetraploid BBCC species. This marker also discriminates $O$. meridionalis from other AA species. Third, Ch04-312W clearly discriminated the EE species, $O$. australiensis and CCDD species from other species. O. longistaminata was discriminated from other AA species by this marker. Additional use of Ch05-070W enabled $O$. grandiglumis to be distinguished from other CCDD species.

Validation by fragment analysis further enabled several genome groups and species to be distinguished (Supplemental Table 1). Ch02-343W made O. longistaminata clearly separable from other AA species, and Ch06-300W and Ch10044G distinguished tetraploid O. punctata from O. minuta (both BBCC).

\section{Discussion}

In this study, we developed the 22 INDEL markers from the public information of over a million of BESs by means of in silico selection and subsequent PCR-based assessments. They are co-dominant markers reliably and rapidly to discriminate all genome types and several species in the genus Oryza.

Phylogenetic analysis of 102 wild accessions also confirmed the reliability of the markers. In this study, O. rufipogon, O. barthii and O. glumaepatula were unable to be separated (Fig. 2). This result is comparable to those of previous studies, in which the AA-genome species lack clear morphological characteristics, except for $O$. longistaminata (reviewed in Vaughan and Morishima (2003)). A sole grouping of $O$. meridionalis accessions is consistent with the results obtained using restriction fragment length polymorphisms (RFLPs) and short interspersed elements (SINEs) (Doi et al. 1995, Wang et al. 1992, Xu et al. 2005). The longistaminata accessions displayed intraspecific amplicon polymorphisms for an INDEL marker (asterisked in Fig. 1), probably representing genetic diversity of this species previously reported (Kiambi et al. 2005, Oka 1988).

$\mathrm{BB}, \mathrm{CC}, \mathrm{BBCC}$ and $\mathrm{CCDD}$ genome species are distributed widely in Asia, Australia, Central and South America and Africa and have been called the "officinalis complex" because of diverse but relatively similar morphology (Tateoka 1962). RFLP analysis in a previous study revealed that these species are closely related (Wang et al. 1992), corresponding 


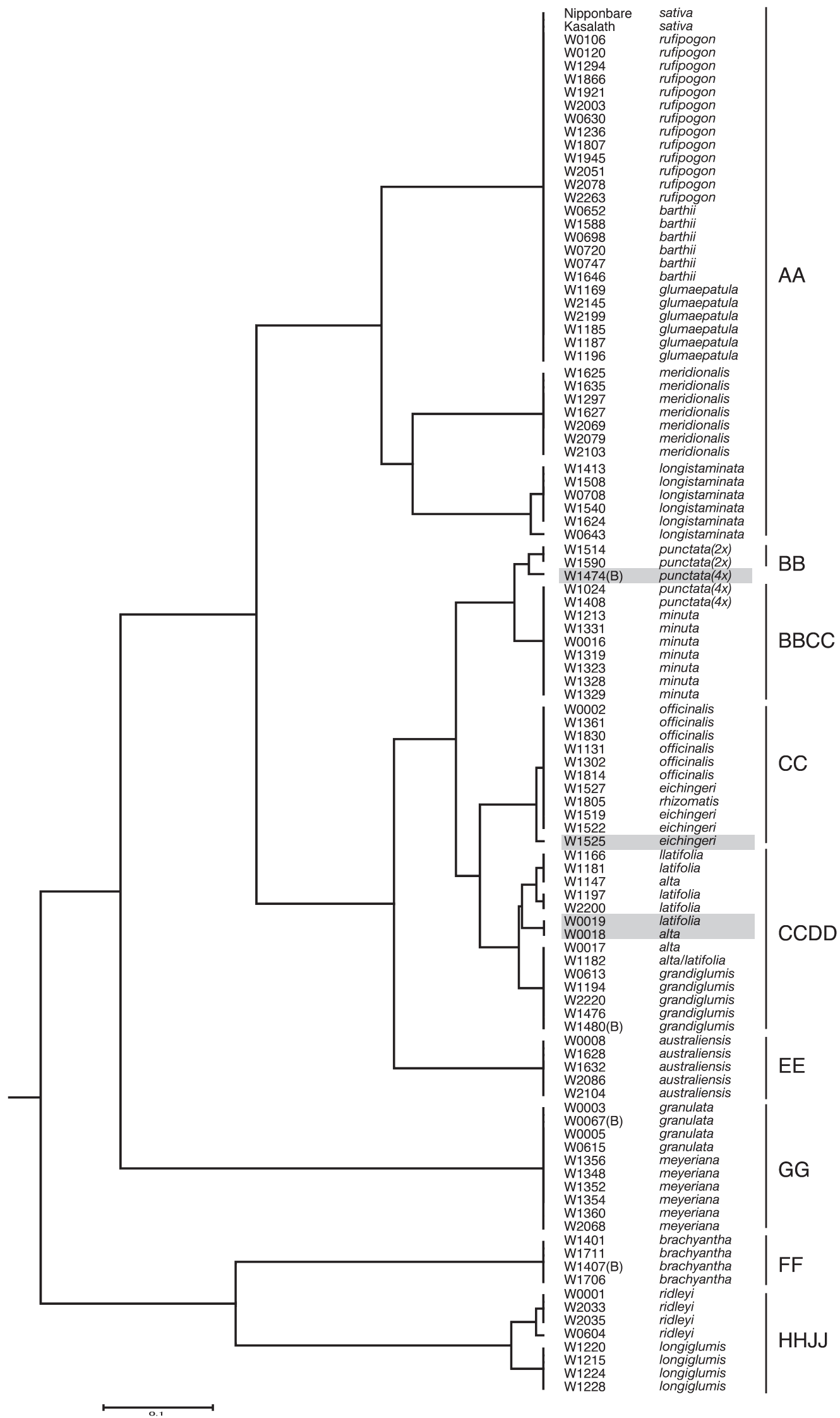

Fig. 2. Phylogenetic tree of 102 wild Oryza accessions and two cultivars obtained using 22 INDEL markers. This phylogenetic tree was depicted by the UPGMA method (Sokal and Michener 1958), based on data shown in Supplemental Table 1. 
to the results of this study. Three CCDD tetraploids, the $O$. latifolia, $O$. alta and $O$. grandiglumis, shared almost INDEL patterns with each other, resulting in all these accessions being in 4 closely-related phylogenetic groups (Fig. 2). This result seems to be consistent to a previous report that these three species are closely related in terms of taxonomy; latifolia and alta were distinguished from each other only by the size of spikelet, and that grandiglumis was distinguished from latifolia and alta only by large glumes (Morishima and Martin 1994). EE genome species were closer to the officinalis complex species than GG, FF and HHJJ species, consistent with a previous proposal in which the EE genome is closely related to the DD genome progenitor ( $\mathrm{Ge}$ et al. 1999, Wang et al. 1992). The phylogenetic results in this study largely corresponded to those in the previous studies, indicating the reliability of the INDEL markers in identification of wild species of rice.

Phylogenetic analysis in this study also revealed possibility of several mistakes in registration of wild accessions conserved in the NIG collection. W1525 was classified as diploid O. eichingeri (CC) and W0018 and W0019 as tetraploid $O$. alta and $O$. latifolia (CCDD), respectively. However, the pattern of PCR amplification by three INDEL markers, Ch07-233W, Ch03-128W and Ch04-312W (Supplemental Fig. 1), suggests the possibility that these three accessions should be classified as tetraploid BBCC species. In addition, W1474, deposited as tetraploid O. punctata (BBCC), exhibited the INDEL pattern of diploid O. punctata (BB) (Supplemental Fig. 1). These problem accessions were involved in the clades distinct from the expected clades in the phylogenetic tree (shaded in Fig. 2). In case of W1525 and W1474, we extended the number of plants examined and confirmed by INDEL marker-assisted and flow-cytometric analyses that each of these accessions actually segregated into two different ploidies (Supplemental Fig. 3). These results clearly demonstrate the reliability of the INDEL markers developed in this study. In the current database, W1805 from Sri Lanka is deposited as $O$. rhizomatis, but was previously deposited as $O$. eichingeri (http://www.shigen.nig.ac.jp/rice/ oryzabaseV4/strain/wildCore/detail/36). However, the haplotype of the 22 INDELs in this accession is identical to that in African $O$. eichingeri accessions (Fig. 2). These problematic accessions need to be reclassified more carefully.

Genetic resources are the common heritage of humankind. However, some natural populations of Oryza species have been lost from their original habitats, mainly due to drastic environmental changes and human activities (Akimoto et al. 1996, Nonomura et al. 2010). In situ and ex situ conservation strategies have become important globaly. Wild Oryza species are quite diverse genetically and physiologically and sometimes form mixed populations of different species in their original habitats. Especially for ex situ conservation, it is critical to pay attention to maintaining the genetic reliability of strains by excluding any contamination from different species. The 22 PCR-based and co-dominant INDEL markers developed in this study will be powerful tools to help determinate species identity and genome types easily and to establish germplasm stocks with corroborative genetic information useful for experimental studies and rice breeding.

\section{Acknowledgments}

We thank Yurie Tamaki and Akemi Ishii for assistance in INDEL evaluation and Sonoko Saeki (NIG) for growing rice plants. We also thank Yukiko Yamazaki, Rie Tsuchiya and Shingo Sakaniwa (NIG) for updating INDEL marker information on Oryzabase. This work was funded by a grant from the National Bioresource Project (NBRP) of the Ministry of Education, Culture, Sports, Science and Technology (MEXT), Japan.

\section{Literature Cited}

Akimoto,M., Y.Shimamoto and H.Morishima (1996) A case study of genetic erosion of wild rice in Thailand. Rice Genet. Newsl. 13: 60-62.

Altschul,S.F., T.L.Madden, A.A.Schaffer, J.Zhang, Z.Zhang, W. Miller and D.J.Lipman (1997) Gapped BLAST and PSI-BLAST: a new generation of protein database search programs. Nucleic Acids Res. 25: 3389-3402.

Ammiraju, J.S., M.Luo, J.L.Goicoechea, W. Wang, D. Kudrna, C. Mueller, J.Talag, H.Kim, N.B.Sisneros, B.Blackmon et al. (2006) The Oryza bacterial artificial chromosome library resource: construction and analysis of 12 deep-coverage large-insert BAC libraries that represent the 10 genome types of the genus Oryza. Genome Res. 16: 140-147.

Doi,K., A.Yoshimura, M.Nakano, N.Iwata and D.A.Vaughan (1995) Polygenetic study of AA genome species of genus Oryza using nuclear RFLP. Rice Genet. Newsl. 12: 160-162.

Ge,S., T.Sang, B.R.Lu and D.Y.Hong (1999) Phylogeny of rice genomes with emphasis on origins of allotetraploid species. Proc. Natl. Acad. Sci. USA 96: 14400-14405.

Jena,K.K. (2010) The species of the genus Oryza and transfer of useful genes from wild species into cultivated rice, $O$. sativa. Breed. Sci. 60: 518-523.

Kiambi,D.K., H.J.Newbury, B.V.Ford-Lloyd and I.Dawson (2005) Contrasting genetic diversity among Oryza longistaminata (A. Chev et Roehr) populations from different geographic origins using AFLP. African J. Biotech. 4: 308-317.

Kottapalli, K.R., M.LakshmiNarasu and K.K.Jena (2010) Effective strategy for pyramiding three bacterial blight resistance genes into fine grain rice cultivar, Samba Mahsuri, using sequence tagged site markers. Biotechnol. Lett. 32: 989-996.

McCouch,S.R., L.Teytelman, Y.Xu, K.B.Lobos, K.Clare, M.Walton, B.Fu, R.Maghirang, Z.Li, Y.Xing et al. (2002) Development and mapping of 2240 new SSR markers for rice (Oryza sativa L.). DNA Res. 9: 257-279.

McCouch, S.R., K. Zhao, M.Wright, C.W.Tung, K. Ebana, M. Thomson, A.Reynolds, D.Wang, G. DeClerck, M.L.Ali et al. (2010) Development of genome-wide SNP assays for rice. Breed. Sci. 60: 524-535.

Michaels, S.D. and R.M.Amasino (1998) A robust method for detecting single-nucleotide changes as polymorphic markers by PCR. Plant J. 14: 381-385. 
Miyabayashi,T., K.I.Nonomura, H.Morishima and N.Kurata (2007) Genome size of twenty wild species of Oryza determined by flow cytometric and chromosome analyses. Breed. Sci. 57: 73-78.

Morishima,H. and P.S.Martin (1994) Investigations of plant genetic resources in the Amazon basin with the emphasis on the genus Oryza-Report of study-tour 1992/93. In: Morishima, H., Y. Shimamoto, Y.Sano and Y.I.Sato (eds.) Reports of the Study-tours for Investigation of Wild and Cultivated Rice Species. Part II., National Institute of Genetics, Japan, pp. 251-357. (The pdf is available on the website; http://www.shigen.nig.ac.jp/rice/oryzabaseV4/ asset/ricereport/Part_II.pdf)

Neff,M.M., J.D.Neff, J.Chory and A.E.pepper (1998) dCAPS, a simple technique for the genetic analysis of single nucleotide polymorphisms: experimental applications in Arabidopsis thaliana. Plant J. 14: $387-392$.

Nei,M., F.Tajima and Y.Tateno (1983) Accuracy of estimated phylogenetic trees from molecular data. J. Mol. Evol. 19: 153-170.

Niizeki,H. and K. Oono (1968) Induction of haploid rice plant from anther culture. Proc. Jpn. Acad. 44: 554-557.

Nonomura,K.I., H.Morishima, T.Miyabayashi, S.Yamaki, M.Eiguchi, T.Kubo and N.Kurata (2010) The wild Oryza collection in National BioResource Project (NBRP) of Japan: History, biodiversity and utility. Breed. Sci. 60: 502-508.

Oka,H.I. (1988) Origin of cultivated rice. J. Sci. Soc. Press/Elsevier, Tokyo.

Pacurar,D.I., M.L.Pacurar, N.Street, J.D.Bussell, T.I.Pop, L.Gutierrez and C.Bellini (2012) A collection of INDEL markers for map- based cloning in seven Arabidopsis accessions. J. Exp. Bot. 63: 2491-2501.

Rogers, S.O. and A.J.Bendich (1988) Plant molecular biology manual. Kluwer Academic Publishers, Dordrecht. pp. 1-10.

Sokal,R.R. and C.D.Michener (1958) A statistical method for evaluating systematic relationships. The Univ. Kansas Sci. Bullet. 38: 1409-1438.

Tateoka,T. (1962) Taxonomic studies of Oryza I. O. latifolia complex. Bot. Mag. Tokyo 75: 418-427.

Vaughan,D.A. and H.Morishima (2003) Biosystematics of the genus Oryza. In: Smith,C.W. (ed.) Rice: Origin, History, Technology, and Production, John Wiley \& Sons, Inc., New York. pp. 27-65.

Wang,Z.Y., G.Second and D.Tanksley (1992) Polymorphism and phylogenetic relationships among species in the genus Oryza as determined by analysis of nuclear RFLPs. Theor. Appl. Genet. 83: 565-581.

Wing, R.A., J.S. Ammiraju, M.Luo, H.Kim, Y.Yu, D.Kudrna, J.L. Goicoechea, W.Wang, W.Nelson, K.Rao et al. (2005) The Oryza Map Alignment Project: the golden path to unlocking the genetic potential of wild rice species. Plant Mol. Biol. 59: 53-62.

Xu,J.H., N.Kurata, M.Akimoto, H.Ohtsubo and E.Ohtsubo (2005) Identification and characterization of Australian wild rice strains of Oryza meridionalis and Oryza rufipogon by SINE insertion polymorphism. Genes Genet. Syst. 80: 129-134.

Yamazaki,Y., S.Sakaniwa, R.Tsuchiya, K.I.Nonomura and N.Kurata (2010) Oryzabase: an integrated information resource for rice science. Breed. Sci. 60: 544-548. 